\title{
Tactical Data Link Communication System Performance in Severe Electromagnetic Environment
}

\author{
Hao Xie, Manxi Wang \\ State Key Lab of Complex Electromagnetic Environment \\ Effects on Electronic and Information System, China, \\ Luoyang, 471003 \\ E-mail: xiehao@coer-zju.org
}

\begin{abstract}
A Tactical Data Link Communication System (TDLCS) simulation model is proposed utilizing the software of Simulink/Matlab, and the TDLCS performances of different parts are compared. Further, such simulator is adopted for evaluating anti-interference performance of the TDLCS for various electromagnetic interference (EMI) signals.
\end{abstract}

Keywords-TDLCS; DSSS; FHSS; EMI; Bit error rate (BER)

\section{INTRODUCTION}

The Tactical Data Link Communication System (TDLCS) [1] is often used for defence purpose. However, the TDLCS always faces with a severe transmission electromagnetic (EM) environment, such as non-intentional and intentional electromagnetic interferences (IEMIs) [2], [3], which may degrade the transmission data quality seriously. So the TDLCS should have good ability to suppress the EMI or IEMI effect, and it is important for us to explore some effective techniques to enhance the TDLCS capability. Furthermore, it is also important to investigate the effect of different kinds of IEMIs on the TDLCS.

In this paper, based on the Simulink platform of Matlab software with the technologies of direct-sequence spreadspectrum (DSSS) [4] and frequency hopping spread-spectrum (FHSS) [5], a TDLCS simulation model is proposed. In addition, communication system performance is improved by using channel coding techniques of cyclic redundancy check (CRC) [6], Reed-Solomon (RS) codes and interleaving [7], [8], [9]. The simulator is successfully used for evaluating the TDLCS performance and the anti-interference performance of the TDLCS to various EMI signals [10], and some bit error rate (BER) figures are plotted according to Simulations.

\section{SPREADING GAIN}

The anti-interference ability of a spread spectrum communication system is stronger than for a conventional communication system. We usually introduce the concept of spreading gain [5] to measure the anti-jamming ability of a spread spectrum communication system. In a spread spectrum system, the spreading gain $G$ is defined as the ratio of de-

\author{
Jun $\mathrm{Hu}$ \\ Centre for Optical and Electromagnetic Research (COER), \\ Zhejiang University, Hangzhou 310058, China \\ E-mail: hujun@zju.edu.cn
}

spread spectrum output SNR of the receiver and the input SNR of the receiver.

$$
G=\frac{S_{0} / N_{0}}{S_{i} / N_{i}}
$$

where $S_{0} / N_{0}$ is the de-spread spectrum output SNR of receiver and $S_{i} / N_{i}$ is the input SNR of receiver.

In a direct sequence spread spectrum system, the spreading gain is equal to the ratio of the pseudo-random sequence spread spectrum signal bandwidth (i.e. sequence spreading code rate $R_{c}$ ) to the information data signal bandwidth (i.e. the information data rate $R_{b}$ ).

$$
G=\frac{R_{c}}{R_{b}}
$$

The bigger spreading gain of the spread spectrum communication system is, the greater immunity to noise interference the system has.

\section{TECHNOLOGY OF TDLCS}

Fig. 1 shows the schematic of the TDLCS. It can perform 20 times DSSS and hops among 32 frequencies points, where the frequency separation of every two neighbouring frequency points is $10 \mathrm{MHz}$.

In order to improve the TDLCS performance of resisting EMI signals in the complex EM environment, some key techniques of spread spectrum and channel coding are applied, such as DSSS, FHSS, CRC, RS and interleaving etc. 


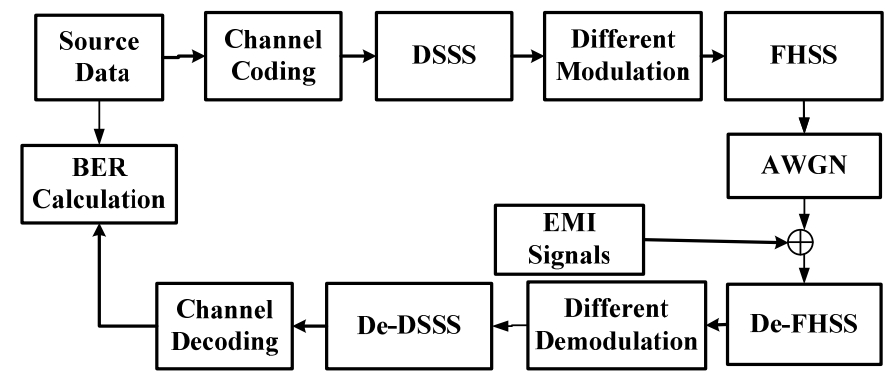

Fig. 1. Schematic of the TDLCS

\section{A. Direct-sequence Spread Spectrum (DSSS)}

DSSS is the spread spectrum technique that is widely used. A DSSS communication system spreads the transmitted signal as Eq. (3) to a wide frequency band by a pseudonoise (PN) code as Eq. (4) [4]. The spread signal takes up more bandwidth than the original signal. At the receiver, the signal is restored to the original one using the same PN code as at the transmitter. The rate of PN sequence $R_{c}$ is much higher than the rate of source one $R_{a}$, and the value of $R_{c} / R_{a}$ is the times of the DSSS, which is an integer.

$$
\begin{aligned}
& a(t)=\sum_{n=0}^{\infty} a_{n} w_{a}\left(t-n T_{a}\right) \\
& c(t)=\sum_{n=0}^{\infty} c_{n} w_{c}\left(t-n T_{c}\right)
\end{aligned}
$$

Figs. 2(a) and 2(b) are frequency spectrogram before and after DSSS respectively. Comparing Figs. 2(a) with 2(b), it is found that the bandwidth after DSSS is 20 times of the bandwidth before DSSS. The simulation model we designed realizes 20 times spread spectrum, and the spreading gain is $10 \log _{10} 20=13 \mathrm{~dB}$. It is seen that the amplitude after DSSS is $13 \mathrm{~dB}$ lower than the amplitude before DSSS. So the spread signal can be hidden in the noise to avoid being detected during the transmission.

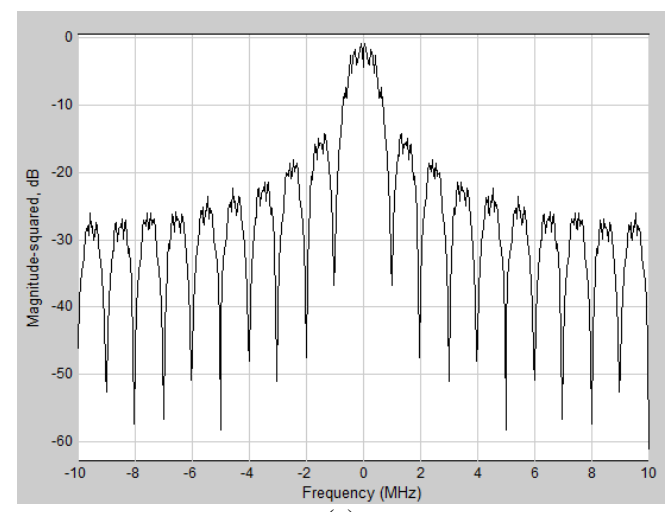

(a)

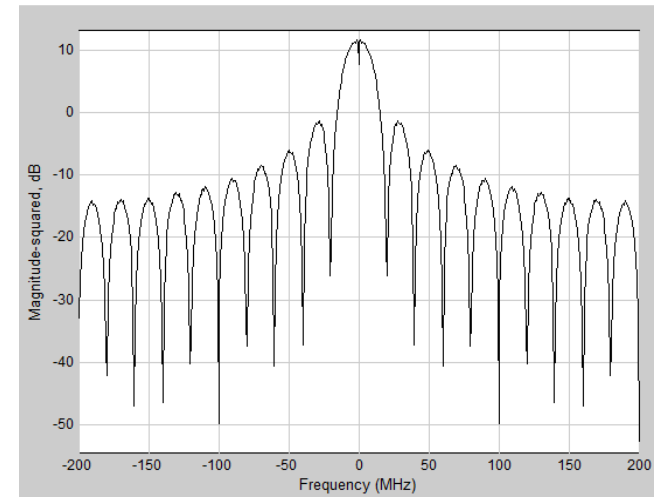

(b)

Fig. 2. frequency spectrogram:

(a) before DSSS (b) after DSSS

\section{B. Frequency-hopping Spread Spectrum (FHSS)}

In the FHSS technology, the carrier frequency of the communication system is changed rapidly and randomly with a PN sequence [5]. The PN sequence is only known by the transmitter and the receiver. The $\mathrm{PN}$ sequence is not transmitted from the transmitter to the receiver directly, but only used for selecting the working frequency with the PN sequence known in advance by the transmitter and receiver.

The transmitted signal $\mathrm{s}(\mathrm{t})$ after frequency-hopping can be expressed as

$$
\mathrm{s}(\mathrm{t})=\mathrm{b}(\mathrm{t}) \cos \left[\left(\omega_{0}+\mathrm{n} \Delta \omega\right) \mathrm{t}+\varphi_{0}\right]
$$

where $b(t)$ is the transmitted signal after modulation, $\cos \left[\left(\omega_{0}+\mathrm{n} \Delta \omega\right) \mathrm{t}+\varphi_{0}\right]$ is the frequency-hopping signal, which is the output one of frequency synthesizer.

At the receiver, the frequency of received signal is recovered using the same $\mathrm{PN}$ sequence as that at the transmitter.

The period of PN sequence is very large and its complexity is very high. So, it is very difficult to detect the PN sequence changing rule by an intentional interferer. Thus, the FHSS communication system cannot be disturbed seriously, and it has high capability for anti- interferences.

It is seen that there are some Gaussian white noise and frequency-hopping signal in Fig. 3(a). Fig. 3(b) is frequency spectrogram of the signal after de-frequency-hopping. The frequency spectrum of single frequency interference and AWGN signal are spread again to the both sides of the recovered center narrow band signal in Fig. 3(b). And the transmitted signals are recovered even single frequency interference signal exists. 


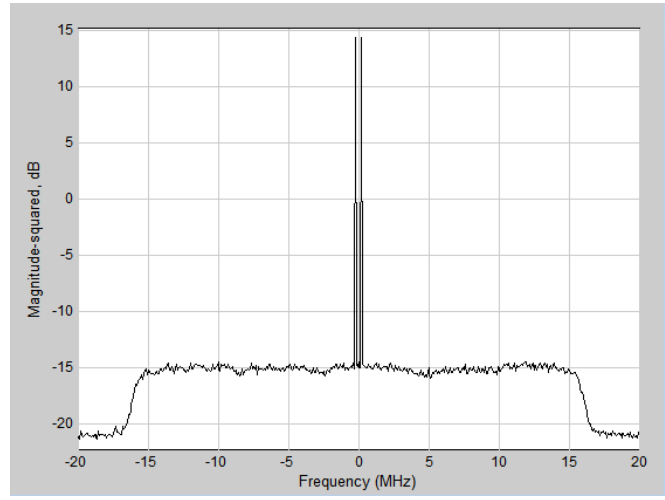

(a)

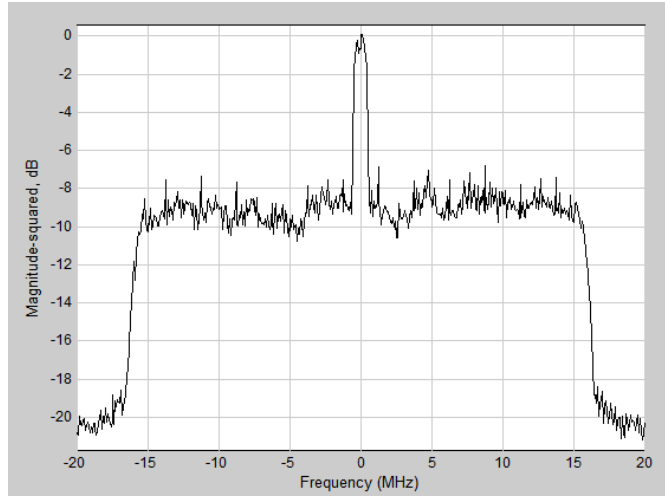

(b)

Fig. 3. frequency spectrogram:

(a) before DE-FHSS (b) after DE-FHSS

\section{Channel Coding}

As we know, some errors often exist during the information transmission because of non-ideal channel and additive white Gaussian noise (AWGN). The system BER can be reduced as some suitable channel coding techniques are used. The channel coding techniques considered here are CRC, RS codes and interleaving.

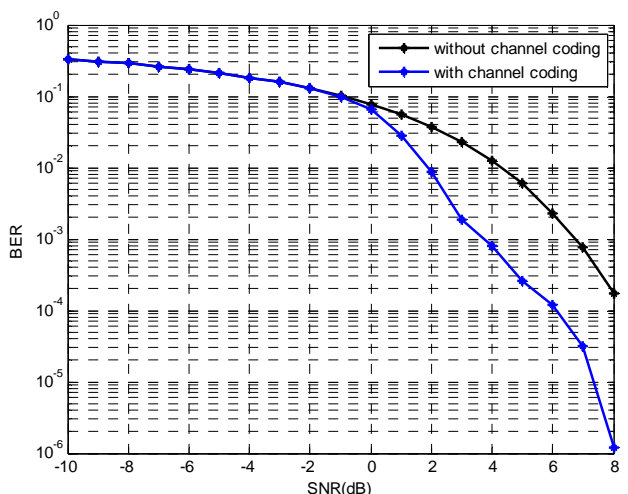

Fig. 4 . BERs of with and without channel coding

Comparing the curves in Fig. 4, it is found that the BER of the blue line is obviously lower than the other when SNR is higher than $0 \mathrm{~dB}$. This result illustrates that the channel coding and decoding parts can improve the system performance effectively.

\section{TDLCS PERFORMANCE}

The modules of MSK, DSSS, FHSS, channel coding and modulation are combined into one simulation model to become the designed TDLCS. We simulate the performance of the TDLCS first.

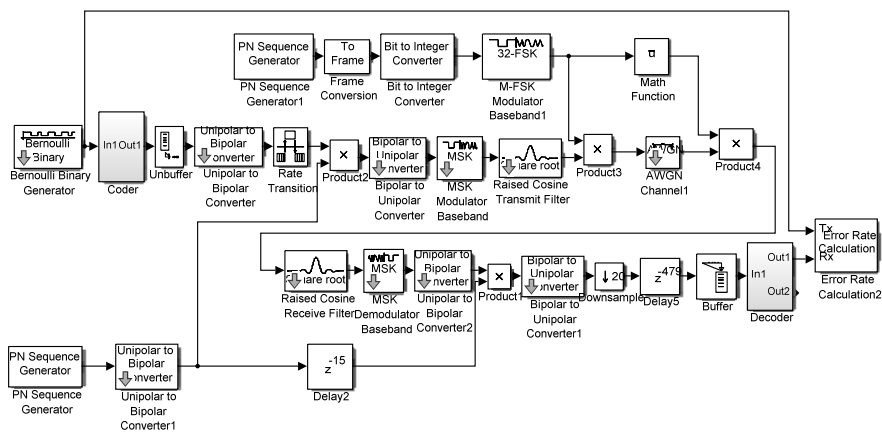

Fig. 5. MSK modulation TDLCS

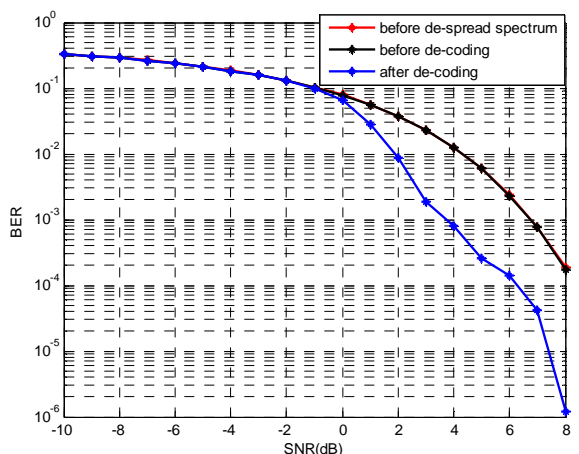

Fig. 6. BER of MSK modulation TDLCS simulation model

We can see from fig. 6 the BER of the blue line is obviously lower than others when SNR is higher than $-1 \mathrm{~dB}$. This result illustrates that the channel coding and decoding parts can improve the system performance effectively. And we also can see from this figure that the BER curves before despread spectrum and before de-coding are almost the same. This is because the noise power suppression capability of the spread spectrum communication system is the ratio of spread spectrum bandwidth to the noise bandwidth, as we discussed in section 2. When the noise bandwidth and the spread spectrum bandwidth are both very wide, the noise power suppression ability of the system is no longer significant. The bandwidth of the AWGN channel module we used here is very wide.

Then add the common EMI signals of PN code MSK modulation jamming, random binary code modulation jamming and noise amplitude modulation jamming we built after the AWGN channel module to investigate the EMI resistance performance of the TDLCS. We can get the below BER figure by changing the jamming signal type. To characterize the jamming influence, we choose MSK modulation and set the parameter signal to noise ratio (SNR) of AWGN channel module to be $100 \mathrm{~dB}$ with the influence of AWGN ignored. 


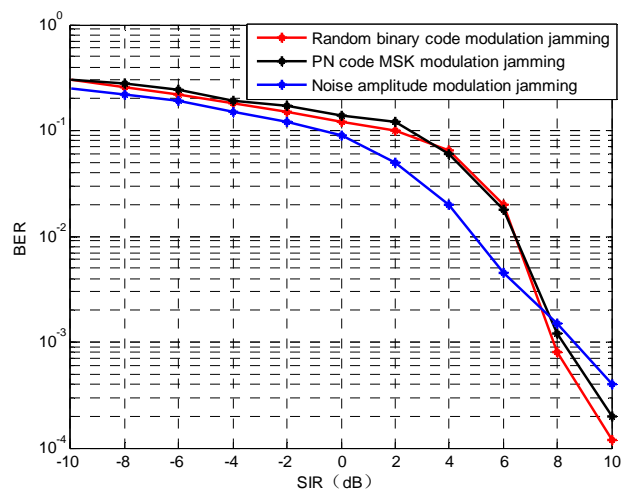

Fig. 7. the BERs of MSK modulation

It can be seen from fig. 7 that the effect of the jamming signals we built to the TDLCS can be described as follows. The jamming effect of the PN code MSK modulation jamming is a little better than the random binary code modulation jamming effect, and the jamming effect of the noise amplitude modulation jamming is the worst. It is also seen that the BER is very low while the SIR is $8 \mathrm{~dB}$, so the modulated signal can be recovered even when the transmission signal is distorted by the jamming signal seriously.

\section{CONCLUSIONS}

In this paper, we have combined the spread spectrum and channel coding techniques into an integrated communication system for building up the TDLCS simulation model. Based on this simulator, the TDLCS performances of different parts are compared. The anti-interference performance of the TDLCS to various EMI signals have also been evaluated, respectively. The BER figures have demonstrated the jamming effects on the TDLCS for different EMI signals.

\section{ACKNOWLEDGMENT}

The work was supported by the State Key Lab of Complex Electromagnetic Environment Effects on Electronic and Information System.

\section{REFERENCES}

[1] W. Z. Wan, J. L. Zhou and P. C. Luo, "Review of tactical data link technology,” Electronics Optical \&Control, vol. 15, pp. 41-46, 2008.

[2] M, Kaur, S. Kakar and D. Mandal, "Electromagnetic interference," International Conference on Electronics Computer Technology , vol. 4, pp. 1-5, 2011.

[3] H. Rong and M. R. Chen, "Influence and Countermeasures on the Shipborne Communication Equipment of Naval Field Complex Electromagnetic Environment,” International Symposium on Distributed Computing and Applications to Business, Engineering \& Science (DCABES), pp. 345-347, 2012.

[4] R. L. Pickholtz, D. L. Schilling and L. B. Milstein, "Theory of SpreadSpectrum Communications-A Tutorial," IEEE Transactions on Communications, vol. 30, pp. 855-884, 1982.

[5] M. B. Pursley, "Direct-Sequence Spread-Spectrum Communications for Multipath Channels," IEEE Transactions on Microwave Theory and Techniques, vol. 50, pp. 653-661, Mar. 2002.

[6] J. Lindholm, "An Analysis of the Pseudo-Randomness Properties of Subsequences of Long m-Sequences," IEEE Transactions on Information theory, vol. 14, pp. 569-576, July 1968.

[7] R. F. Pawula and R. F. Mathis, “A Spread Spectrum System with Frequency Hopping and Sequentially Balanced Modulation--Part I: Basic Operation in Broadband Noise,” IEEE Transactions on Communications, vol. 28, pp. 682-688, May 1980.

[8] W. W. Peterson and D. T. Brown, "Cyclic Codes for Error Detection,” Proceedings of the IRE, vol. 49, pp. 228-235, Jan. 1961.

[9] J. YIN, Y. L. YIN and J. Z. XU, "Simulation Analysis of CRC-RS Coding in JTIDS,” Communications Technology, vol. 435, pp. 24-29, 2010.

[10] J. Chen and P. Owsley, “A Burst-Error-Correcting Algorithm for ReedSolomon Codes,” IEEE Transactions on Information Theory, vol.38, pp. 1807 - 1812, Nov. 1992. 